\title{
Comparison of Two Kinds of Endurance Training Programs on the Effects of the Ability to Recover in Amateur Soccer Players
}

\author{
Slavko Rogan ${ }^{1,2,}$ \\ ${ }^{1}$ Department of Health, Discipline Physiotherapy, Bern University of Applied Sciences, Bern, Switzerland \\ 2 Academy for Integrative Physiotherapy and Training Education (AfiPT), Grenzach-Wyhlen, Germany \\ ${ }^{*}$ Corresponding author: Slavko Rogan, Department of Health, Discipline Physiotherapy, Bern University of Applied Sciences, Bern, Switzerland. Tel: +41-318483536, Fax: +41-318483521, \\ E-mail: slavko.rogan@bfh.ch
}

Received: August 8, 2014; Revised: September 15, 2014; Accepted: September 25, 2014

\begin{abstract}
Background: High intensity intermittent aerobic exercise is an elementary endurance training exercise to build soccer endurance. Many studies exist with professional soccer players. But limited research has been conducted with amateur soccer players.

Objectives:The aim of this study was to compare and assess the effects of the shuttle-run method and the Hoff-track method on the ability to recover in amateur soccer players within three weeks.

Patients and Methods: Two amateur soccer teams were randomly assigned to shuttle-run group ( $\mathrm{n}=24$; SRG) (SRG: shuttle-run group) or Hoff-track group ( $\mathrm{n}=18$; HTG) (HTG: hoff-track group). They performed 2 times/week over three weeks their program. SRG performed a $20 \mathrm{~m}$ high speed shuttle-run until exhaustion and HTG covered at their highest speed level an obstacle track. Before and after training the yo-yo intermittent recovery test level 2 (YYIRTL2) was conducted.

Results: Significant differences were observed within $(\mathrm{P}<0.05)$ and between the groups $(\mathrm{P}=0.06$; $\mathrm{ES}=0.50)$ in distance covering during YYIRTL2.

Conclusions: Both training methods seem to improve the ability to recover in amateur soccer players within a short time period during the competition season.
\end{abstract}

Keywords: Soccer; Physical Endurance; Physical Fitness

\section{Background}

For field position, team success, and competition level, soccer players need an optimal combination of technical and tactical skills (1), physical performance (2), physical characteristics (e.g. somatotypes) (3) and mental motivation (2). Not only in professional soccer, but also increasingly in amateur soccer, economic constraints are exposed. For this reason, even amateur clubs are interested in constantly adapting to the latest developments. This includes research methods, which provide current training methods and laboratory or field tests for evaluating physical performance and performance skills. Monitoring is necessary to estimate the effectiveness of training sessions and for optimizing physical performance and soccer skills. The scientist has not only to spread knowledge in professional soccer, but also to draw attention to amateur soccer (4).

Soccer, a stop-and-go sport (5) is described as a noncyclical, random game, which is often interrupted by breaks $(3,6)$. Running is a predominant activity in soccer. Professional male soccer players cover between 9800 and 12500 $\mathrm{m}$ at an average intensity close to the anaerobic lactate threshold, or 80 - 90\% of maximal heart rate frequency during the game, or being $70-80 \%$ of their maximal oxygen uptake $\left(\mathrm{VO}_{2 \max }\right)(6)$. Amateur soccer players do not differ significantly from professional soccer players in $\mathrm{VO}_{2 \max }(7,8)$, but differ significantly in $\mathrm{VO}_{2}$ kinetic (7) and total distance covered during the Yo-Yo Intermittent Recovery Test level 2 (YYIRTL2) (8). More crucial for professional and amateur soccer players is the distance coverable with maximal high-intensity running during a game (9) and a rapid ability to recover between intensive intermittent exercises (4).

High-intensity interval training (HIT) at maximal aerobic speed or supra-maximal aerobic speed (shuttle run) has been developed to improve $\mathrm{VO}_{2 \max }$ that increases aerobic endurance performance. The literature described that HIIT induces greater improvements in aerobic and anaerobic capacity compared with continuous training (10). However, several soccer coaches believe that this kind of exercise is not specific to soccer because there is no soccer technical component. Hoff et al. (11) designed a soccer specific track. This track allows simulating actions of soccer skills such as dribbling, changing action with the ball and also physical fitness.

Reduced endurance training during the season has negative effect on match endurance performance (abil- 
ity to recover) during the competition season. It is known that aerobic fitness significantly decreases from midseason onward in amateur soccer players (12). Therefore, it is important to maintain or to improve endurance performance (ability to recover) during the whole season in amateur soccer players. Stolen et al. (6) described that soccer teams should perform one short period of endurance training in between the two halves of the season. Many studies were conducted in professional soccer players. Such studies are rare in amateur soccer (4).

\section{Objectives}

The aim of this study was to compare high intensity intermittent aerobic training programs for the ability to recover in amateur soccer players during competition season. Two methods were compared: on the one hand the shuttle-run method without a ball and on the other hand the Hoff-track methods, where players have to dribble a ball through an obstacle track. The question arises; is it possible to improve endurance performance during three weeks and which method can better improve endurance performance in amateur soccer players?

\section{Patients and Methods}

\subsection{Study Design}

A two-group pretest-posttest design was used. Two teams were randomly assigned to a shuttle-run group (SRG) or to a Hoff-track group (HTG).

\subsection{Participants}

This study examined two amateur German soccer teams with 24 males (sixth division, mean age: $25.1 \pm 4.4$ years) and with 18 males (eighth division, mean age: $25.3 \pm 4.6$ years). Inclusion criteria were: minimum of eight years of training and weekend match day experience, trained at least two days per week for an average of 90 minutes each time, and members of the same soccer team. Exclusion criteria: current injuries or missed $20 \%$ of training sessions. This study is consistent with the ethical requirements for human experimentation in accordance with the latest version of the Declaration of Helsinki and the institution proofed the study protocol. The soccer players gave their written informed consent.

\subsection{Yo-Yo Intermittent Recovery Test level 2}

To test the ability to recover, the Yo-Yo Intermittent Recovery Test level 2 (YYIRTL2) was applied (13). The YYIRTL2 consisted of $20 \mathrm{~m}$ shuttle runs performed at increasing velocities with ten seconds of active recovery between runs until exhaustion. Audio cues of the YYIRTL2 were recorded on a CD (The Yo-Yo tests, bangsbosport.com) and broadcast using an Apple MacBook Pro (Apple, Cupertino, USA) and connectable boxes (Ion, Cumberland, USA). The end of the test was considered to be when the soccer player twice failed to reach the front line in time (objective evaluation) or he felt unable to complete another shuttle at the dictated speed (subjective evaluation). The total distance covered during the YYIRTL2 (including the last incomplete shuttle) was considered to be the test score for ability to recover.

\subsection{Intervention Program}

All players performed a high intensity intermittent aerobic exercise program two times a week over three weeks. The SRG performed a $20 \mathrm{~m}$ high speed shuttlerun until exhaustion. Between the runs a 10-second active recovery rest was carried out. The HTG had to cover as much distance as possible at their highest speed level while dribbling a ball over an obstacle track (Figure 1) for 4 minutes over 4 sets and with a 4 minute active recovery break between sets (11). Hurdle height was set at $0.5 \mathrm{~m}$ and training cone used in the dribbling circuit were 0.3 $\mathrm{m}$ high and $0.15 \mathrm{~m}$ wide. Working intensity was set at $90 \%$ at maximum running speed with active recovery break of jogging at $30-40 \%$ of maximum running speed.

\subsection{Statistical Analysis}

Analysis was carried out using IBM SPSS Version 21 (SPSS Inc., Chicago, Illinois, USA) and Microsoft Excel 2007 (Microsoft Corporation, Redmond, USA). For the descriptive statistical processing, means and standard deviation were calculated. For comparison of the endurance performance data paired sample t-test and independent sample t-test were used. The level of significance for all statistical procedures was defined at $\mathrm{P} \leq 0.05$. Effect size (ES) was calculated to assess meaningfulness of differences. Effect sizes were considered to be $>0.8=$ large, 0.8-0.5 $=$ moderate,$<0.5-0.2=$ small, and $<0.2=\operatorname{trivial}(14)$.

Figure 1. Hoff Track

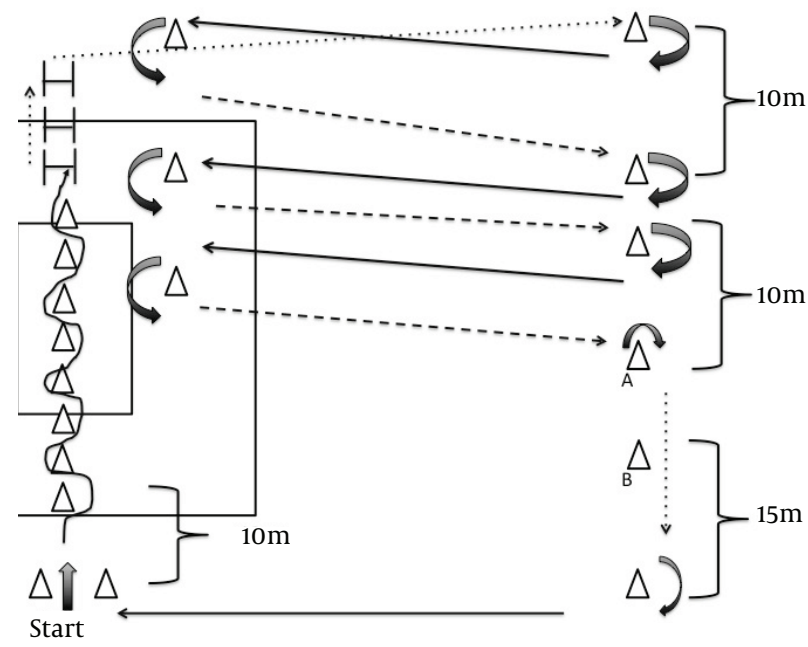

The ball is dribbling in the direction of the arrows. Between cone A and cone B backwards running, $\Delta$ : Cones; $\longmapsto$ : Hurdles 
Table 1. Descriptive Statistics of the Yo-Yo Intermittent Recovery Test Level 2 in Meters a,b,c

\begin{tabular}{|c|c|c|c|c|c|}
\hline Variable & Pre-Test, $\mathrm{m}$ & P Value Between & Post-Test, m & P Value Within ES ${ }^{\mathrm{d}}$ & P Value Between ES $\mathrm{e}^{\mathrm{e}}$ \\
\hline Yo-Yo group $(n=24)$ & $438.33 \pm 149.66$ & & $644.33 \pm 273.97$ & $0.001 \pm 0.49$ & \\
\hline Hoff-group $(n=18)$ & $358.33 \pm 131.61$ & 0.79 & $434.44 \pm 148.67$ & $0.001 \pm 0.46$ & $0.006 \pm 0.50$ \\
\hline
\end{tabular}

\section{Results}

No drop out could be determined. Table 1 shows the mean (SD) of the YYIRTL2 variables. Significant differences were observed within $(\mathrm{P}<0.05)$ and between the groups $(\mathrm{P}=0.06 ; \mathrm{ES}=0.50)$.

\section{Discussion}

The goal of this study was to compare the effects of the shuttle-run and Hoff-track methods on ability to recover in amateur soccer players during competition season. This current study shows that it's possible to improve running distance in the SRG by up to $206 \mathrm{~m}$ (+ 31.97\%; ES $=0.49)$ and in the HTG by up to $76.11 \mathrm{~m}(+11.3 \%$; ES = 0.46) covered during the YYIRT2 by high intensity intermittent aerobic exercise methods two times a week over three weeks in amateur soccer players. These types of exercise show similar responses that occur during a soccer game. This would be a decrease in phosphorylcreatine, muscle $\mathrm{pH}$ adenosine triphosphate (ATP) and high contribution of anaerobic and aerobic pathways.

After high intensity intermittent aerobic exercise the body adapts with central factors such as higher $\mathrm{VO}_{2 \max }$ on the basis of changes such as heart and blood volume and peripheral mechanisms such as elevation of mitochondrial enzymes, activity of lactate dehydrogenase 1-2 isozymes and an elevated number and size of mitochondria (13). A consequential result is a better ability to recover based on enlarged heart stroke volume (15) and on better muscle metabolism with decreased glycolysis activation and in increased efflux of lactate to the blood (16). Tesch and Wright (17) described a relation between capillary density in the muscle and blood lactate concentration. This implies that a higher capillary density reduces the distance between the capillary and muscle fiber. Hence faster oxygen delivery and more efficient removal of metabolites can occur. This study is in accordance with previous investigations (18) that described improvements in distance covering after high intensity intermittent aerobic exercise.

It is known that specific nutritional recommendations are required to optimise performance during training and game and improve and accelerate recovery and optimise the body weight (19). The amateur soccer players have not changed their lifestyle habit. They went to their usual occupation ( $<8$ hours per day), the nutrition was the same and the sleep rhythm was retained. In this current study anthropometrics were not measured after the intervention period. Body composition is an important factor where body weight must be moved against gravity (20). Decrease in body fat shows better sprint performance, because body fat acts as dead weight during performance. In future studies body fat measurements should be taken into account.

\section{Acknowledgements}

Thanks to all players for their effort and thanks Ross Bennie for proof reading.

\section{Financial Disclosure}

The article I have submitted to the journal for review is original, has been written by the stated authors and has not been published elsewhere. The article is not currently being considered for publication by any other journal and will not be submitted for such review while under review by this journal. The article contains no libelous or other unlawful statements and does not contain any materials that violate any personal or proprietary rights of any other person or entity.

\section{References}

1. Rampinini E, Impellizzeri FM, Castagna C, Coutts AJ, Wisloff U. Technical performance during soccer matches of the Italian Serie A league: effect of fatigue and competitive level. J Sci Med Sport. 2009;12(1):227-33.

2. Bangsbo J. The physiology of soccer-with special reference to intense intermittent exercise. Acta Physiol Scand Suppl. 1994;619:1-155.

3. Rogan S, Hilfiker L, Clarys P, Clijsen R, Taeymans J. Position-specific and team-ranking-related morphological characteristics in German amateur soccer players - a descriptive study. Int J Appl Spor Sci. 2011;23(1):168-82.

4. Rogan S, Wust D, Ringgenberg M, Sager R, Schmidtbleicher D. Comparison of endurance performance and stretch-shortening cycle between two male amateur soccer teams - a cross-sectional pilot study. Asian J Sports Med. 2014

5. Rogan S, Wust D, Schwitter T, Schmidtbleicher D. Static stretching of the hamstring muscle for injury prevention in football codes: a systematic review. Asian J Sports Med. 2013;4(1):1-9. 
6. Stolen T, Chamari K, Castagna C, Wisloff U. Physiology of soccer: an update. Sports Med. 2005;35(6):501-36.

7. Rampinini E, Sassi A, Azzalin A, Castagna C, Menaspa P, Carlomagno D, et al. Physiological determinants of Yo-Yo intermittent recovery tests in male soccer players. Eur J Appl Physiol. 2010;108(2):401-9.

8. Wells CM, Edwards AM, Winter EM, Fysh ML, Drust B. Sport-specific fitness testing differentiates professional from amateur soccer players where VO2max and VO2 kinetics do not. J Sports Med Phys Fitness. 2012;52(3):245-54.

9. Krustrup P, Mohr M, Amstrup T, Rysgaard T, Johansen J, Steensberg A, et al.The yo-yo intermittent recovery test: physiological response, reliability, and validity. Med Sci Sports Exerc. 2003;35(4):697-705.

10. Gorostiaga EM, Walter CB, Foster C, Hickson RC. Uniqueness of in terval and continuous training at the same maintained exercise intensity. EurJ Appl Physiol Occup Physiol. 1991;63(2):101-7.

11. Hoff J, Helgerud J. Endurance and strength training for soccer players: physiological considerations. Sports Med. 2004;34(3):165-80.

12. Magal M, Smith RT, Dyer JJ, Hoffman JR. Seasonal variation in physical performance-related variables in male NCAA Division III soccer players. J Strength Cond Res. 2009;23(9):2555-9.
13. Bangsbo J, Mohr M, Poulsen A, Perez-Gomez J, Krustrup P. Training and testing the elite athlete.J Exerc Sci Fit. 2006;4(1):1-14.

14. Cohen J. Statistical Power Analysis for the Behavioral Sciences. 2 ed. New Jersey: Lawrence Erlbaum Associates; 1988.

15. Cooper G. Basic determinants of myocardial hypertrophy: a review of molecular mechanisms. Annu Rev Med.1997;48:13-23.

16. Fox EL, Bowers R, Foss M. The physiological basis of physical education and athletics.California:Wm. C. Brown; 1989.

17. Tesch PA, Wright JE. Recovery from short term intense exercise: its relation to capillary supply and blood lactate concentration. Eur J Appl Physiol Occup Physiol.1983;52(1):98-103.

18. Impellizzeri FM, Marcora SM, Castagna C, Reilly T, Sassi A, Iaia FM, et al. Physiological and performance effects of generic versus specific aerobic training in soccer players. Int J Sports Med. 2006;27(6):483-92.

19. Garcia-Roves PM, Garcia-Zapico P, Patterson AM, IglesiasGutierrez E. Nutrient intake and food habits of soccer players: analyzing the correlates of eating practice. Nutrients. 2014;6(7):2697-717.

20. Casajus JA. Seasonal variation in fitness variables in professional soccer players. J Sports Med Phys Fitness. 2001;41(4):463-9. 